\title{
Study on the evaluation of the clinical effects of traditional chinese medicine in heart failure by complex intervention: protocol of SECETCM-HF
}

\author{
Jingyuan Mao*1, Yazhu Hou², Hongcai Shang2, Henghe Wang1, \\ Xianliang Wang ${ }^{1}$, Yingqiang Zhao ${ }^{3}$, Tianfu Niu ${ }^{4}$, Jinrong Cui ${ }^{5}$, \\ Guangping Li ${ }^{6}$, Qian Lin 7 , Le Shi ${ }^{8}$, Xiuli Jia9 ${ }^{9}$ Ruihong Fan ${ }^{10}$, Baohe Wang2, \\ Hongwu Wang ${ }^{2}$ and Jishou Ruan ${ }^{11}$
}

\begin{abstract}
Address: ${ }^{1}$ Cardiovascular Department of The First Teaching Hospital of Tianjin University of Traditional Chinese Medicine, 314 Anshan Western Road, Nankai District, Tianjin, China, 2Tianjin University of Traditional Chinese Medicine, 312 Anshan Western Road, Nankai District, Tianjin, China, ${ }^{3}$ Cardiovascular Department of The Second Teaching Hospital of Tianjin University of Traditional Chinese Medicine, 816 Zhenli Road, Hebei District, Tianjin, China, ${ }^{4}$ Shanxi Traditional Chinese Medicine Institute, 46 Bingzhou Western Street, Taiyuan, Shanxi Province, China, ${ }^{5}$ The Integrated Hospitals of TCM and Western Medicine of Shanxi University of Traditional Chinese Medicine, 13 Fudong Street, Taiyuan, Shanxi Province, China, ${ }^{6}$ The Second Teaching Hospital of Tianjin Medical University, 23 Pingjiang Road, Hexi District, Tianjin, China, ${ }^{7}$ The Eastern Hospital of Beijing University of Traditional Chinese Medicine, 6 No.1 District Fangxing Yuan, Fang Village, Fengtai District, Beijing, China, ${ }^{8}$ Tianjin Beichen TCM Hospital, Jinjing Road, Beichen District, Tianjin, China, ${ }^{9}$ Tianjin Nankai TCM Hospital, 28 No.2 Guangkai Street, Nankai District, Tianjin, China, ${ }^{10}$ Tianjin TCM Hospital, 354 Beima Road, Hongqiao District, Tianjin, China and ${ }^{11}$ Nankai University, 94 Weijin Road, Nankai District, Tianjin, China

Email: Jingyuan Mao* - jymao@126.com; Yazhu Hou - mreleven@163.com; Hongcai Shang - shanghongcai@126.com; Henghe Wang - henghewang@126.com; Xianliang Wang - xlwang1981@126.com; Yingqiang Zhao - zhaoyingqiang1000@126.com; Tianfu Niu - niutianfu2584@sina.com; Jinrong Cui - cjinrong21417@sina.com; Guangping Li - tjcardiol@126.com; Qian Lin - linqian@mail.cintcm.ac.cn; Le Shi - xfile01@sina.com; Xiuli Jia - xiulijia123@163.com; Ruihong Fan - fanruihong6399@yahoo.com.cn; Baohe Wang - wbh3423@sina.com; Hongwu Wang -wanghw55@tjutcm.edu.cn; Jishou Ruan - jsruan@nankai.edu.cn

* Corresponding author
\end{abstract}

Published: 24 December 2009

Trials 2009, 10:122 doi:10.1186/1745-6215-10-122

This article is available from: http://www.trialsjournal.com/content//0/I/I22

(C) 2009 Mao et al; licensee BioMed Central Ltd.

This is an Open Access article distributed under the terms of the Creative Commons Attribution License (http://creativecommons.org/licenses/by/2.0), which permits unrestricted use, distribution, and reproduction in any medium, provided the original work is properly cited.
Received: 8 October 2009

Accepted: 24 December 2009

\begin{abstract}
Background: Experts in Traditional Chinese Medicine (TCM) have studied the TCM subject of the pathogenesis of heart failure (HF) for several decades. As a result, the general idea is ben deficiency and biao excess. However, the clinical evaluation system which combined the TCM and western medicine in HF has not been developed yet. The objective is to establish the evaluation index system for the integration of TCM and western medicine. The evaluation indexes which include TCM items will specify the research design and methods.
\end{abstract}

Methods: Nine medical centers in different cities in China will participate in the trial. A population of 340 patients with $\mathrm{HF}$ will be enrolled through a central randomized system for different test groups. Group A will be treated with only western medicine, while group B with western and Chinese medicine together. The study will last for 12 months from the date of enrollment. The cardiovascular death will be the primary outcome.

Discussion: By putting the protocol into practice, the clinical effects of TCM for HF will be identified scientifically, objectively as well as rationally. The proper index system which built in the study will be helpful for the clinical effect expression of HF by integrated medicine in future.

Trial Registration: ChiCTR-TRC-00000059 


\section{Background}

Ancient TCM literatures have records of successful treatment methods for heart failure. In modern times, medical experts have recognized the general idea of heart failure in TCM pathogenesis as ben deficiency and biao excess [1,2]. They have revealed the multi-mechanisms of its efficiency of HF as well [3]. Nowadays, lots of intravenous and oral preparations have been applied in treating HF, and confirmed that they're effective [4-7]. However, the formations of projects involving randomized clinical trials in TCM are recent and their designs are not as rigorous as the clinical trials in western medicine. In this way, neither the conclusion which defined by death nor the special clinical effects of TCM in HF was revealed properly. This restricted the application and development of TCM in HF deadly.

\section{Objective}

In order to evaluate the clinical effects of heart failure and establish a clinical effect expression of HF treated with integrated medicine, the protocol of SECETCM-HF will be implemented. It was strictly designed under the law of randomized controlled trial (RCT).

\section{Methods \\ Design}

The multi-centre randomized controlled trial will be practiced in 9 hospitals in Tianjin, Beijing and Shanxi Province of China. The trial begins on September $17^{\text {th }} 2008$.

\section{Ethical Aspects}

The trial was approved by the Ethics Committee of First Teaching Hospital of Tianjin University of TCM on January the $30^{\text {th }}, 2008$ (TYLL2008004). It's registered in the Chinese Clinical Trial Registry and the International Clinical Trials Registry Platform of WHO as well. The protocol and its informed consent form had been judged by the Committee to be ethically and scientifically satisfactory to the aims. Written informed consent must be obtained from all participants or their representatives before enrolling.

\section{Patients}

According to the inclusion and exclusion criteria, totally 340 cases with HF will be randomized into two groups, that are Group A (regular medication group, as comparison) and Group B (regular medication + TCM syndrome differentiation group).

Inclusion criteria are:

- Chronic heart failure patients (The medical history of primary cardiovascular diseases; symptoms and signs typical of heart failure; $L V E F \leq 50 \%$ ).

- Cardiac function classification: New York Heart Association (NYHA) II IV.
- Aged 40-79.

- Completed and submitted informed consent form.

Exclusion criteria are:

- Those whose Brain Natriuretic Peptide (BNP) $<200$ $\mathrm{pg} / \mathrm{mL}$

- Those who suffer from acute heart failure.

- Those who have one of the following disease: 1) acute myocardial infarction(within the previous 4 weeks); 2) pulmonary heart disease; 3) severe valve disease; 4) hypertrophic obstructive cardiomyopathy; 5) congenital heart disease; 6) pulmonary hypertension which was caused by acute or chronic pulmonary embolism or other reasons; 7) pre-excitation syndrome; 8) stroke within 6 months; 9) acute myocarditis.

- Those who suffer from sever hepatic or renal deficiency.

- Those who have severe endocrine diseases like hyperthyroidism.

- Those who suffer from severe anemia.

- Those who have mental disease.

- Those women who are pregnant or during the lactation period.

- Those who participated in other studies within the last two months.

\section{Western medication}

According to the guidelines for chronic heart failure $[8,9]$, the diuretics, angiotensin converting enzyme inhibitor (ACEI) or angiotensin receptor blocker (ARB), $\beta$-blockers, aldosterone receptor antagonist, digitalis and vasodilating agents could be used as the regular medication in participants. The hospitalization period is $2 \pm 1$ weeks.

\section{Trial treatment}

The program for Group A is as follows (Fig. 1):

Based on the regular medication, the medicines like polarized solution (500 ml, Qd), which are considered as neutral treatment and the same fluid volume to Group B, can be used intravenously during the hospitalization period. After discharging, two kinds of placebo drugs, which are visually identical to Group B, should be selected and prescribed for 6 months according to the differentiation of $q i$ yang or qi-yin syndrome (Table 1). For the patients who 
suffer from qi-yang deficiency (combined with blood stasis and/or phlegm retention), the placebo drug of Qiliqiangxin Capsule should be taken. Conversely, the placebo drug of Buyiqiangxin tablets can be applied in those with qi-yin deficiency (combined with blood stasis and/or phlegm retention). Only one kind of them will be taken 4 pieces each time and 3 times a day.

The program for Group B is as follows (Fig. 1):

Based on the regular medication mentioned above, during the hospitalization period, the patients who are qiyang deficient can take Shenfu Injection $(60 \mathrm{ml})$ with $5 \%$ glucose $(250 \mathrm{ml})$ intravenously guttae once daily. For those who are qi-yin deficient, Shenmai Injection $(60 \mathrm{ml})$ with $5 \%$ glucose $(250 \mathrm{ml})$ can be applied intravenously guttae once daily. For both, Danhong Injection $(40 \mathrm{ml})$ will be used with 5\%glucose or physiological saline (250 $\mathrm{ml}$ ) intravenously guttae once daily. After discharging, different drugs should be selected for 6 months according to the differentiation of qi-yang or qi-yin. For the patients who suffer from qi-yang deficiency (combined with blood stasis and/or phlegm retention), Qiliqiangxin Capsule should be taken. Conversely, Buyiqiangxin tablets can be applied in those with qi-yin deficiency (combined with blood stasis and/or phlegm retention). Only one of them will be taken 4 pieces each time and 3 times a day.

Shenfu injection (Batch number: 081202), Shenmai injection (Batch number: 081125) and Danhong Injection (Batch number: 090410) which are used in the hospitalized period are made in Yaan Sanjiu Medical and Pharmaceutical CO. LTD, Chiatai Qingchunbao Pharmaceutical CO. LTD and Buchang Group separately. The Qiliqiangxin Capsule (Batch number: 080601), Buyiqingxin Tablets (Batch number: 080701) their models (Batch number: 080701 and M090101 separately) are supported by Hebei Yiling Pharmaceutical CO. LTD and Suzhou Zilu Pharmaceutical CO. LTD freely.

\section{Follow-up}

The observing period is 12 months, which includes a 6month experimental medication period and a 6-month follow-up period. At admission, discharging and each follow-up point (Fig. 2), each patient's general data and their four diagnostic results in TCM should be recorded (Table 2 ). During the observing period, other herbs with noted cardiac effects are forbidden for prescription or personal

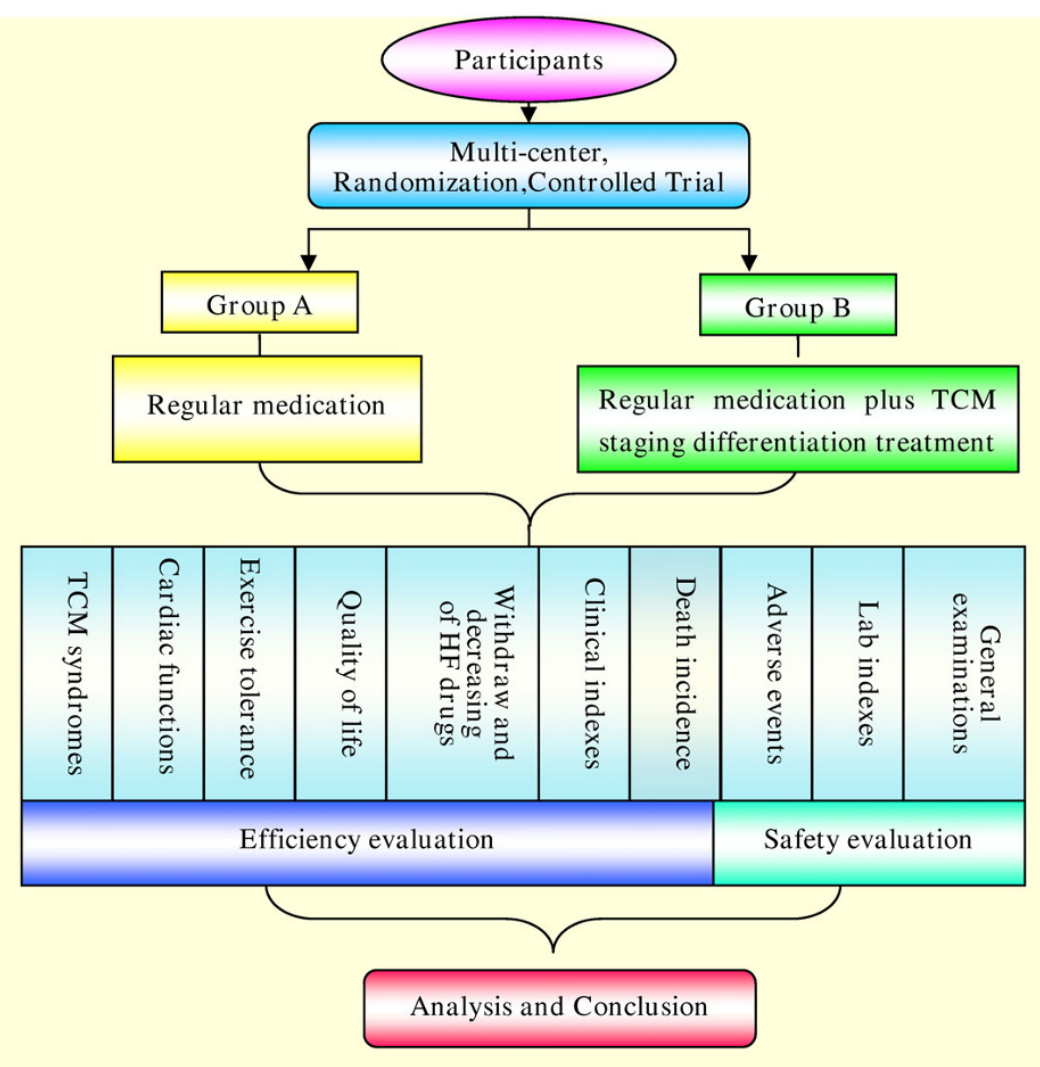

Figure I

The General Process of SECE-TCM. 
use. The patients should be followed-up for 12 months, except the dead ones.

\section{Primary outcome measurement}

The primary outcome measure is "cardiovascular death incidence".

\section{Secondary outcome measurement}

The following secondary outcome measures will be assessed:

- HF re-admission.

- Other cardiovascular events: including acute coronary syndrome, serious cardiac arrhythmias, cardiac shock, stroke, pulmonary embolism and peripheral vascular incidences.

- Non-cardiovascular events: including upper gastrointestinal hemorrhage, kidney failure and tumor.

\section{Measures of health-related quality of life}

The Modified Minnesota Quality of Life Scale consists of the items of its own and the additional items with TCM characteristics. It's one of the special features in the study, and used as an evaluation principle for HF fulfilled with Chinese culture elements.

\section{Measures of safety}

From admission to the $3^{\text {rd }}$ and $6^{\text {th }}$ month interval during the follow-up period, regular examinations should be provided. The vital signs of the patients, such as the heart rate and blood pressure, should be recorded before and after treatment. Blood and urine routine examination, hepatic and renal function, as well as electrolytes in blood should also be tested. Moreover, ECG should be recorded at each follow-up period. The adverse events, especially the severe ones, should be reported to the leader of the hospital and drug administration.

\section{Data collection}

Clinical research format should be completed at each follow-up point by the investigators. All patients enrolled will be recorded.

The statisticians from the statistics center in Tianjin University of Traditional Chinese Medicine and the mathematics experts from Nankai University are responsible for the statistic process and data mining.

\section{Data collection instruments}

The patients should be evaluated at the points of admission, discharging, and the $1^{\text {st }}, 3^{\text {rd }}, 6^{\text {th }}, 9^{\text {th }}, 12^{\text {th }}$ month after leaving hospital (Fig. 2). The items below should be measured:

- NYHA classification.

- Lee's heart failure score

- Six-minute walking test (6MWT)

- The scoring of the four diagnosis of TCM

- Quality of life scale

- The withdrawal and decreasing of HF drugs.

- General examination in clinic, including cardiac ultrasound, chest radiography, ECG, the plasma level of angiotensin and aldosterone, blood and urine routine examination, hepatic and renal function, as well as electrolytes in blood.

- Inflammatory cytokines (TNF- $\alpha$, IL-1, IL-6) and BNP

\section{Sample size}

The fatality rate of patients with HF in China is about $24.24 \%$ [10] when they are prescribed medication includ-

Table I: The Standardization of TCM Differentiation.

\begin{tabular}{ll}
\hline & Qi yang deficiency \\
\hline Main symptoms & Palpitation, shortness of breath, tired and fatigue \\
\hline Secondary symptoms & $\begin{array}{l}\text { Spontaneous perspiration, aversion to cold and cold limbs, anorexia and } \\
\text { abdominal distension, loose stool, soreness and lumbago, dizziness and amnesia, } \\
\text { pale complexion, dark pale tongue or pale and corpulent tongue with teeth prints, } \\
\text { deep slow or irregularly intermittent pulse. }\end{array}$ \\
Combined syndromes red zygoma, red tongue with \\
$\begin{array}{l}\text { I. Blood stasis: Dull pain on the chest, cyanotic lips, raised jugular venous pressure, dark or purple tongue with petechia, } \\
\text { and hesitant pulse. } \\
\text { 2. Phlegm retention: Swelling face and limbs or other parts of body, oliguria, chest fullness, cough with sputum, white and } \\
\text { slippery tongue coating }\end{array}$
\end{tabular}

P.S. If the patient has the main symptom, two or more secondary symptoms with the proper tongue and pulse characters, and either one or two item of the combined symptoms, he/she can be diagnosed as heart failure disease with certain TCM syndrome. 
Table 2: Definitions used in SECETCM-HF.

\begin{tabular}{|c|c|}
\hline Term & Definition \\
\hline Differentiation & $\begin{array}{l}\text { By TCM diagnostic method, to differentiate patients' TCM syndrome } \\
\text { comprehensively, and then determine the treating principles based on it. }\end{array}$ \\
\hline Qi Deficiency & Insufficiency of vital $Q i$ or dysfunction of $Q i$ \\
\hline Yin Deficiency & Insufficiency of essence, blood or body fluid \\
\hline Yang Deficiency & Insufficiency or dysfunction of yang $Q i$ \\
\hline Blood Stasis & Unsmooth blood circulation \\
\hline Phlegm Retention & $\begin{array}{l}\text { Concrete or invisible pathological products caused by dysfunction of body fluid } \\
\text { metabolism }\end{array}$ \\
\hline Cardiac Ultrasound Indexes & Using improved-Simpson method to calculate ESV, EDV and EF, et al. \\
\hline The information obtained by TCM four diagnostic methods & $\begin{array}{l}\text { The items of TCM syndromes, tongue and pulse characters by inspecting, listening to } \\
\text { the sound and smelling the odors, inquiring and pulse-taking. }\end{array}$ \\
\hline Quality of Life & $\begin{array}{l}\text { Evaluating the quality of life by Minnesota Quality of Life scale combined with TCM } \\
\text { characteristic items }\end{array}$ \\
\hline
\end{tabular}

ing diuretics, ACEI or ARB, $\beta$-blockers and vasodilating agent. In a retrospective study of the First Teaching Hospital of Tianjin University of TCM, the rate was below $10 \%$. The power calculation is based on the fatality rates at the $5 \%$ significance level. There should be 140 patients in each group. Concerning the loss to follow-up rate, the study needs 340 patients in all.

\section{Method of randomization}

The central randomization system makes the study possible. The trial center gives the unique enrollment number of each participant, then records and preserves the information after randomization. Each hospital could assign only 1 to 2 investigators as contact person to send the patients' information to the central randomization system, and get feedback on the patient's random group.



\section{Figure 2}

The Implementation of SECE-TCM.

\section{Statistical analyses}

For the statistical analysis, both medical statistic method and complex mathematical methods will be used. The case-distribution, commeasurable analysis, patients' compliance, therapeutic effect analysis, security analysis and correlation analysis are determined by the statistical method. Focusing on the occurrence and corresponding time of the cardiovascular events, product-limit method, Log-Rank test and Kaplan-Meier curve will be applied. Meanwhile, the total risk rate will be analyzed by the Cox proportional hazard regression. The complex mathematical methods include the rough set, random graph and support vector machine.

\section{Discussion}

On the increasing demand of traditional medicines (including TCM), the investigators should find a way to 
express the characteristic clinical effects, and make it overspread worldwide in a sustainable way. In 1995, the report of Office of Alternative Medicine (OAM) of American mentioned that "the efficiency evaluation is the core problem" in traditional medicine [11].

Through literature review, experts consultation and epidemiological survey, the investigators have made some essential conclusions which involved information obtained by TCM diagnostic method, syndrome types of HF and the optimized TCM medication program (TCM staging differentiation treatment) $[12,13]$. The information gives supports for the protocol making and evaluation index system building.

In the implementation process of the Study on the Evaluation for the Clinical Effects of Traditional Chinese Medicine in Heart Failure (SECETCM-HF), the regular medication program follows the modern guideline of $\mathrm{HF}$. The systematic and comprehensive evaluation information from clinical observing and follow-up are collected to abstract the clinical evaluation indexes. Not only death event, re-hospitalization and the general modern lab indexes, but also the TCM information will be included in the evaluation indexes system. In order to make the clinical effect predominance of TCM expressed properly, all information will be analyzed by both medical statistical and complex mathematical methods which make the establishment of integrated TCM and western medicine evaluation index system possible.

\section{Abbreviations}

SECETCM-HF: Study on the Evaluation of the Clinical Effects of Traditional Chinese Medicine in Heart Failure; TCM: Traditional Chinese Medicine; HF: Heart failure; RCT: Randomized controlled trial; NYHA: New York Heart Association; BNP: Brain Natriuretic Peptide; ACEI: Angiotensin converting enzyme inhibitor; ARB: Angiotensin receptor blocker; ECG: Electrocardiogram; 6MWT: Six-minute walking test; OAM: Office of Alternative Medicine

\section{Competing interests}

Each author has participated sufficiently in the work to take public responsibility for appropriate portions of the content. All authors read and approved the final manuscript and declare no competing interests.

\section{Authors' contributions}

JYM made substantial contributions to the conception and design of the study and took charge of drafting the study protocol essentially. HCS was responsible for the evaluation on the endpoints as well as adverse events of the study and participated in the methodological design. YQZ, YZH, XLW, HHW, TFN, JRC, GPL, QL, LS, XLJ and
RHF took part in the coordination of the trial. BHW was in charge of the supervising of the trail. HWW took charge of the statistics design and the statistical analyses. JSR dealt with the mathematic management. All authors read and approved the final manuscript.

\section{Acknowledgements}

Study on Evaluation for the Clinical Effects of Traditional Chinese Medicine to Heart Failure (SECETCM-HF) is one of the National Key Technology R\&D Programs in the I th Five-Year Plan of China (NO. 2006BAI08B0201). Thanks for the following coordination hospitals: The First Teaching Hospital of Tianjin University of Traditional Chinese Medicine, The Second Teaching Hospital of Tianjin University of Traditional Chinese Medicine, Shanxi Traditional Chinese Medicine Institute, The Integrated Hospitals of TCM and Western Medicine of Shanxi University of Traditional Chinese Medicine, The Second Teaching Hospital of Tianjin Medical University, The Eastern Hospital of Beijing University of Traditional Chinese Medicine, Tianjin Beichen TCM Hospital, Tianjin Nankai TCM Hospital and Tianjin TCM Hospital.

\section{References}

I. Rui Li: The Discussion on TCM Pathogenesis of Congestive Heart Failure. Modern Journal of Traditional Chinese Medicine and Pharmacy 2003, I:13-14.

2. Mianhua Wu: Pathogenesis of Congestive Heart Failure. Journal of Nanjing University of Traditional Chinese Medicine 200I, 17:206-209.

3. Xiaojie Lu, Fayuan Lu, et al:: The Research Progress of Mechanism on Heart Failure by Herbs. Information on Traditional Chinese Medicine 2007, 24:13-15.

4. Huimin Gu: The Clinical Effects of $\mathbf{3 0}$ Ptients by Shenmai Injection on Heart Failure. Forum on traditional Chinese Medicine 2006, 7:40-4I.

5. Hui Li, Xiang Liu, et al:: The clinical effects of Shenfu Injection on Heart Failure. Journal of Emergency in Traditional Chinese Medicine 2002, 8:27I-272.

6. Bo Chen, Hong Lin, et al:: The clinical research of Buyiqingxin Pill on Chronic Heart Failure. Chinese Journal of Geriatric Care 2005, 3:43-45.

7. Huimin Su: The influence on Qulity of Life of Heart Failure Patients by Qiliqingxin Capsule. Chinese Journal of Integrative Medicine on Cardio-Cerebrovascular Disease 2007, 20:917-918.

8. Chinese Medical Association for Heart Disease, The Editorial Committee of Chinese Journal of Cardiology: Guideline for Diagnosis and Treatment on Heart Failure. Chinese Journal of Cardiology 2007, I 2: 1076-1095.

9. Paulus WJ, Tschöpe C, Sanderson JE, Rusconi C, Flachskampf FA, Rademakers FE, Marino P, Smiseth OA, De Keulenaer D, LeiteMoreira AF, Borbély A, Édes I, Handoko ML, Heymans S, Pezzali N, Pieskel B, Dickstein K, Fraser AG, Brutsaert DL: How to diagnose diastolic heart failure:a consensus statement on the diagnosis of heart failure with normal left ventricular ejection fraction. European Heart Journal Advance Access 2007:4.

10. Ruiqin Xie, Jun Du, Yuming Hao: The Obseraion on Coronary Artery Disease Heart Failure by Trimetazidine. Chinese Journal of Cardiology 2004, 32:77.

II. Levin JS, Glass TA, Kushi LH, Schuck JR, Steele L, Jonas WB: Quantitative methods in research on complementary and alternative medicine. A methodological manifesto. NIH Office of Alternative Medicine. Med Care 1997, 35:1079-1094.

12. Xiaolei Cui, Jingyuan Mao, Xianliang Wang, et al.: Analysis of Experts Questionnaire for TCM Pattern of Heart Failure. Acta Universitatis Traditionis Medicalis Sinensis Pharmacologiaeque Shanghai 2009, 23:3I-33.

13. Xiaolei Cui, Jingyuan Mao, Xianliang Wang, et al.: The application of Delphi Method on Selecting TCM Diagnostic Items in Heart Failure. Liaoning Journal of Traditional Chinese Medicine 2009, 36:508-509. 